\title{
Dynamics and Trends in Content System Formation of Nautical Charts
}

\author{
Anastasia Gorevalova ${ }^{\text {a,* }}$, Tamara Vereshchaka ${ }^{\text {a }}$ \\ ${ }^{a}$ Moscow State University of Geodesy and Cartography (MIIGAiK) 1st Author - a.gorevalova@gmail.com, 2nd Author - \\ cartography@miigaik.ru \\ * Corresponding author
}

Keywords: Marine Cartography, Nautical Charts, Paper Navigation Charts and Electronic Charts, Structure and Content of Nautical Charts, Map Symbols

\begin{abstract}
:
Nautical Charts belong to special marine geographic charts designed primarily to aid navigation. Among the marine charts, they hold a unique position in terms of their practical application - they are used for laying a course and determining its location in the sea. Such charts are issued by the national hydrographic offices of the states as part of the national navy.
\end{abstract}

The paper examines over a century-long evolution of the nautical charts content based on the Tables of Symbols published in 1910, 1937, 1961, 1971, 1985 and 2016. The latest 2016 edition is currently in use.

The collection of objects forming the basis of the Charts' content within the period under review remained generally unchanged, with slight fluctuations - with a tendency for increase at the beginning and a tendency for reduction recently. The greatest changes concerned the image of the land part in accordance with the symbols in the topographical maps.

The content of the marine part of the charts was expanded, as a rule, by means of including new sections and developing the existing sections related to the navigational equipment, services and stations. The examples are provided in the general table summarizing the whole period under research and categorizing the objects taken from different sections of the published guidelines.

The analysis of the nautical charts content carried out in this paper allows us to claim that the map symbols are able to provide the necessary informational capacity for the nautical charts designed to aid safe navigation. The informational potential of the charts is ensured by a substantial number of sections containing information about the objects, phenomena, processes which form the features of the marine and land environment as well as the shoreline interacting with waves, swash, high and low tides with different types of foreshores and shore.

In general, the tables of symbols issued in different years are the fruition of years of work as they form a corpus of the symbols formed by incremental accumulation and modification within specific objects or groups of objects. Nowadays, the prevailing trend is the alignment of the navigation data to organize an informational database for an automated creation of digital and electronic charts.

The following trends in the charts content formation within the period under study may be called the most prominent:

- $\quad$ significant changes in the cartographic objects content for the land part in accordance with the topographic symbols - tendency for extension in the first editions and tendency for reduction in the past years. This trend is vividly shown at the coastline representation;

- a considerable increase in the objects corpus, including addition of new sections related to the marine part. This tendency is especially conspicuous in the magnetic elements, navigational equipment (radio aids and radar aids, lights and buoy), services and stations (harbor office, customs office, post office, sound signalling, magnetic stations etc.). There has been a major extension in the marine areas and borders section: from 3 objects in the 1937 edition to 33 objects in the current edition;

- almost all the sections are directly or indirectly connected with the information on the dangers such as relief features, morphological features of banks and riverbeds, port structures, bottom patches and currents. There is a separate "Dangers" section which contains 35 symbols.

- $\quad$ structural alignment of symbols in the latest editions of the tables mainly associated with the symbols publication for the international nautical charts and the adoption of the S-101 product specification for Electronic Navigational Charts. A more efficient arrangement of the mapping objects into sections has been an 
important step forward as compared to the previous S-57 standard, which presents the objects in alphabetical order and therefore makes it difficult to find them in the catalogue.

Despite the positive trends, there are still grave disadvantages in the structure and content of the map symbols, which point out the necessity of further improvement in the future editions.

It is difficult to analyse the information displayed within a single edition due to the fact that the symbols for one class of objects are placed into different sections of the tables; components of different geographic spheres are included into a single section; one and the same cartographical tool used to characterize different objects (cape elevation benchmark, island elevation benchmark, ground point elevation benchmark, peak elevation benchmark, etc.) is presented in the tables as a number of separate symbols. Such examples (given in the complete text of the article) prove the necessity of further alignment and improvement of the charts content.

It is hoped that due to a wider use of electronic maps and database formation, the optimization of the map symbols will be continued with scientific evidence.

A retrospective view of the informational potential in the nautical charts and its evolution throughout an extensive period of time allows to formulate the principal directions in the improvement of the nautical charts structure and content:

- $\quad$ observance and development of the systemic approach principles and use of scientific evidence in the creation of the symbols and map content in general;

- $\quad$ unifying the system of symbols based on the fundamental scientific research in the displayed objects classifications with regard to the current requirements of a versatile use of nautical charts in alignment with the universal topographic maps as well as the international standards for map content;

- $\quad$ maintaining tradition in the representation of the objects specific for the Russian charts while aligning the map symbols with the international standards, in which these symbols may be absent;

- implementation of the projects on the charts improvement along with their update powered by new technologies.

The research was carried out within the state assignment of Ministry of Science and Higher Education of the Russian Federation No 0708-2020-0001. 